\title{
Editorial
}

\section{Magnetism at the Atomic Scale}

\author{
Verónica Salgueiriño $^{*}, 1, \S$ and Juan J. de Miguel $^{2}$
}

\author{
${ }^{1}$ Dpto. de Física Aplicada, Universidade de Vigo, 36310, Vigo, Spain \\ ${ }^{2}$ Dpto. Física de la Materia Condensada and Institute of Materials Science "Nicolás Cabrera", Univ. Autónoma de Madrid, \\ Cantoblanco, 28049-Madrid, Spain
}

The term Nanoscience has been coined to describe the study of materials with sizes of the order of nanometers, reaching down to the limit of even just a few atoms. The emergence of this very broad and multidisciplinary field has been made possible by the advances in both theoretical methods and experimental techniques allowing to explore and manipulate matter at the shortest scales; nanometer-sized systems provide a privileged playground to explore the relationship between the materials' structure and their properties. Research in this area is already provoking a revolution in many areas of industry, fueled by the perspectives of achieving artificial materials with special functionalities. Indeed, when the dimensions of objects become comparable to or smaller than the characteristic lengths associated to the diverse properties, these latter can become substantially altered with respect to the bulk material.

The ferromagnetism of transition metals is a prototypical example of a cooperative phenomenon, arising from the exchange interaction among conduction electrons. It is therefore also extremely sensitive to structural parameters such as the atomic arrangement, the number of neighbours or the interatomic distances (dictated for example by specific interfaces). Indeed, many magnetic properties are known to depend on the details of the atomic environment, the dimensionality or the object's shape. The magnetic moment per atom, for instance, is a sensitive function of atomic coordination: as the overlap between the electronic orbitals from neighbouring atoms diminishes, the density of states at the Fermi level becomes narrower and the imbalance between the spin-up and spin-down sub-bands increases, resulting in an enhanced magnetic moment. Superparamagnetism is another characteristic phenomenon linked to small dimensions; as the number of magnetic moments in a solid and their mutual interactions are reduced, the disordering effect of thermal fluctuations dominates over exchange coupling and the macroscopic magnetization fluctuates spontaneously. This behaviour is also related to the concept of magnetic anisotropy, or the existence of energetically preferred directions for the

*Address correspondence to this author at the Dpto. de Física Aplicada, Universidade de Vigo, 36310, Vigo, Spain; Tel: +34 986 812295; Fax: +34 986 814069; E-mail: vsalgue@uvigo.es

${ }^{\S}$ Guest Editor alignment of the magnetic moments. Deriving from the spinorbit coupling, this property is thus directly related to the spatial arrangement of the constituent atoms within the crystal lattice, which in turn becomes affected by the presence of surfaces or interfaces, among others. The large surface-to-bulk ratios characteristic of nanoparticles are also responsible at least in part for the novel magnetic properties found in these systems.

This sensitivity of magnetism with respect to the structural and morphological parameters creates many opportunities for tailoring the properties of nanomagnets, but at the same time calls for a very careful fabrication and characterization work; defects and inhomogeneities can often play a dominant role in determining the behaviour and performance of materials and devices. The purpose of this Hot Topic Issue is to provide a broad overview of different nanofabrication methods and materials and their resulting magnetic properties. Different types of systems are covered: from nanomagnet arrays fabricated on solid surfaces (Albrecht and Makarov; Neumann et al.; Vázquez et al.) to sets of magnetic nanoparticles obtained by colloidal chemistry synthesis (Pichon et al., Zhang et al.). These latter ones are of particular interest for their potential applications in bio-medicine; nevertheless, their fabrication methods raise some issues that need to be addressed, such as preventing agglomeration, determining the influence of the capping layers or controlling the surface chemistry in a biological environment. Nanomagnet arrays produced on solid surfaces are being thoroughly investigated for their possible uses as data storage media or high-sensitivity sensors. In these cases, the lack of uniformity in the arrangement and characteristics of the nanomagnets is one of the basic problems.

The onset of superparamagnetism when the particles' dimensions drop below a certain limit is a general and rather fundamental question in all these systems; while in some cases it may be desirable -as for the nanoparticles used in hyperthermia treatments-, for other applications such as data storage it represents a very undesirable drawback. This phenomenon is addressed in detail in the article by Serantes and Baldomir, using theoretical and numerical simulation techniques.

In summary, we intend with this special Hot Topic issue of The Open Surface Science Journal to offer an ample and informative perspective of the ongoing work along different lines on this exciting and timely field of research.

(C) Salgueiriño and de Miguel; Licensee Bentham Open.

This is an open access article licensed under the terms of the Creative Commons Attribution Non-Commercial License (http: //creativecommons.org/licenses/by$\mathrm{nc} / 3.0 /$ ) which permits unrestricted, non-commercial use, distribution and reproduction in any medium, provided the work is properly cited. 